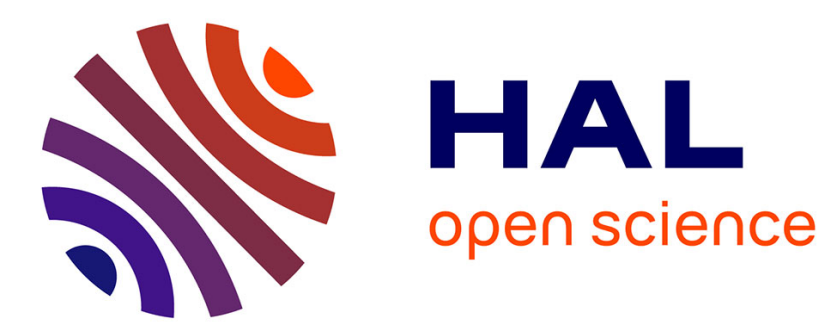

\title{
Contact Detection and Physical Interaction for Low Cost Personal Robots
}

\author{
Fabrizio Flacco, Abderrahmane Kheddar
}

\section{To cite this version:}

Fabrizio Flacco, Abderrahmane Kheddar. Contact Detection and Physical Interaction for Low Cost Personal Robots. RO-MAN: Robot and Human Interactive Communication, Aug 2017, Lisbon, Portugal. lirmm-01363954v2

\section{HAL Id: lirmm-01363954 https://hal-lirmm.ccsd.cnrs.fr/lirmm-01363954v2}

Submitted on 26 Jun 2017

HAL is a multi-disciplinary open access archive for the deposit and dissemination of scientific research documents, whether they are published or not. The documents may come from teaching and research institutions in France or abroad, or from public or private research centers.
L'archive ouverte pluridisciplinaire HAL, est destinée au dépôt et à la diffusion de documents scientifiques de niveau recherche, publiés ou non, émanant des établissements d'enseignement et de recherche français ou étrangers, des laboratoires publics ou privés. 


\title{
Contact Detection and Physical Interaction for Low Cost Personal Robots
}

\author{
Fabrizio Flacco and Abderrahmane Kheddar
}

\begin{abstract}
We present a methodology for estimating joints torque due to external forces applied to a robot with large joints backlash and friction. This undesired non-linearity is common in personal robot, due to the use of low cost mechanical components and type of usage. Our method enables contact detection and human-robot physical interaction capabilities without using extra sensors. The effectiveness of our approach is shown with experiments on a Romeo robot arm from SoftBank Robotics.
\end{abstract}

\section{INTRODUCTION}

One of the challenging vision for the robotics community is to bring a robot in every home for daily assistance and other services [1]. Personal robots, that can help in everyday housework can have a considerable economical and societal impact for the well-being of frail, handicapped or ageing persons. In such application contexts, personal robots must have high cognitive and interactive skills but must also be safe in order to interact in close contact and physically collaborate with humans.

To reach this ambitious goal, different research laboratories and companies are developing new personal robots (e.g. [2], [3], [4]). The trend we observe is toward the utilization of intrinsic robot safety, using lightweight and compliant structures, and also low cost mechanical components, to reduce the cost and make these robots accessible to a large public market.

From the control side, one would like to borrow methodologies developed for industrial robots as they have proven to be reliable in long-time usage. But often, undesired mechanical effects, due to low cost components or intrinsic safety choices, do not allow getting the results obtained in high-precision expensive robots. This is the case of the momentum-based residual signal. This well-known method that has been used for detecting contacts [5], distinguish between unforeseen (collisions) and intentional contacts [6], estimate contact forces [7] and physical interaction control [8]. The peculiarity of the residual method is to allow safe physical human-robot collaboration without using torque

The authors are with Centre National de la Recherche Scientifique (CNRS) and the University of Montpellier (UM), Laboratoire d'Informatique de Robotique et de Microéectronique de Montpellier (LIRMM), Interactive Digital Human (IDH) group, Montpellier, France.

A. Kheddar is also with the CNRS-AIST Joint Robotics Laboratory (JRL), UMI3218/RL,Tsukuba, Japan.

This work is supported by the ROMEO 2 project, (www.projetromeo.com), bpifrance in the framework of the Structuring Projects of Competitiveness Clusters (PSPC) and the Japan Society for Promotion of Science (JSPS) Grant-in-Aid for Scientific Research (B) No. 16H02886, "Cutting Edge Multi-Contact Behaviors". sensing, which makes it an appealing characteristic for low cost personal robots.

Consider the classical robot dynamic model

$$
\boldsymbol{M}(\boldsymbol{q}) \ddot{\boldsymbol{q}}+\boldsymbol{c}(\boldsymbol{q}, \dot{\boldsymbol{q}})=\boldsymbol{\tau}_{M}+\boldsymbol{\tau}_{\mathrm{ext}},
$$

where $\boldsymbol{M}(\boldsymbol{q})$ is the robot inertia matrix, $\boldsymbol{c}(\boldsymbol{q}, \dot{\boldsymbol{q}})=\boldsymbol{C} \dot{\boldsymbol{q}}+\boldsymbol{g}(\boldsymbol{q})$ includes the centrifugal, Coriolis term $\boldsymbol{C}(\boldsymbol{q}, \dot{\boldsymbol{q}}) \dot{\boldsymbol{q}}$, and the gravity effects $\boldsymbol{g}(\boldsymbol{q})$. Where $\boldsymbol{C}(\boldsymbol{q}, \dot{\boldsymbol{q}})$ is built from Cristoffel symbols of second kind. $\boldsymbol{q}, \dot{\boldsymbol{q}}$ and $\ddot{\boldsymbol{q}}$ are the vector of the $n$ joint generalized coordinates and its first and second time derivative, respectively ${ }^{1}$. Finally, $\boldsymbol{\tau}_{M}$ is the vector of motors torque, and $\tau_{\text {ext }}$ is the vector of joints torque due to external contact forces.

The knowledge of the robot dynamic model, and the measurement of $\boldsymbol{q}, \dot{\boldsymbol{q}}$ and $\boldsymbol{\tau}_{M}$ enables the computation of the so-called residual vector [5]

$$
\boldsymbol{r}(t)=K_{i}\left[\boldsymbol{M} \dot{\boldsymbol{q}}-\int_{0}^{t}\left(\boldsymbol{\tau}_{M}+\boldsymbol{C}^{T} \dot{\boldsymbol{q}}-\boldsymbol{g}+\boldsymbol{r}(s)\right) d s\right]
$$

where $K_{i}>0$ is the residual gain, and $\boldsymbol{M} \dot{\boldsymbol{q}}$ is the generalized angular momentum of the robot. By evaluating the time derivative of $\boldsymbol{r}$, and exploiting the well known relation $\dot{M}=\boldsymbol{C}+\boldsymbol{C}^{T}$, it is straightforward to show that the residual $\boldsymbol{r}$ is a filtered version of $\boldsymbol{\tau}_{\text {ext }}$

$$
\dot{\boldsymbol{r}}=K_{i}\left[\boldsymbol{\tau}_{\mathrm{ext}}-\boldsymbol{r}\right]
$$

ideally, $K_{i} \rightarrow \infty \Rightarrow r=\tau_{\text {ext }}$. Thus, the residual can be used for estimating the 'perturbation' of the robot joints due to external contact forces. The residual estimator is characterized by the single parameter $K_{i}$, which is the $-3 \mathrm{~dB}$ bandwidth of the low-pass filtered reconstruction of $\tau_{\text {ext }}$. In fact, when rewriting eq. (3) in the Laplace domain we obtain the unitary first-order filter

$$
\boldsymbol{r}(s)=\frac{1}{1+\frac{1}{K_{i}} s} \boldsymbol{\tau}_{\mathrm{ext}}(s)
$$

with time constant $1 / K_{i}$ equal to the inverse of the filter bandwidth. Then, using the gain $K_{i}$ it is possible to control the bandwidth of the residual. With large $K_{i}$ the signal will be more reactive, but also more sensitive to noise. By lowering $K_{i}$, it is possible to filter the noise, but the response will be slower.

The residual method is effective if the dynamic model is sufficiently accurate. Small errors in the dynamic model

\footnotetext{
${ }^{1}$ In the remaining of the paper we get rid of the dependence to the generalized coordinates (e.g. we write $\boldsymbol{M}$ instead of $\boldsymbol{M}(\boldsymbol{q})$ ) as much as possible
} 
identification can be filtered by using a small threshold on the residual, so as to consider the estimated external torque only when it is beyond the fixed threshold. On the contrary, other unmodeled effects, ordinary when low cost components are employed, do not allow using the residual method in its current (classical) form. Among the unmodeled effects, we consider the joint backlash as illustrated in Sec. II.

The main contribution of our work is in extending the residual method for contact detection considering backlash (and friction) effects. We propose a novel approach to identify the coefficients that characterize joint backlash and friction, using a variation of the residual method (Sec. III). How to implement the residual for estimating joint torque due to external contacts with the new model is presented in Sec. IV. In Sec. V, we present experimental results using the prototype of the left arm of Romeo robot from SoftBank Robotics, showing: i) the benefits with respect to the classical residual; ii) the effectiveness of contact detection both with the robot at a given static posture or in motion; and iii) the use of our residual extension to allow physical robot manipulation and collaboration.

\section{JOINT BACKLASH}

Joint backlash is an undesired effect of joint mechanisms; it is usually due to a play in the transmission between the motor shaft and the link shaft, as illustrated in Fig. 1. The

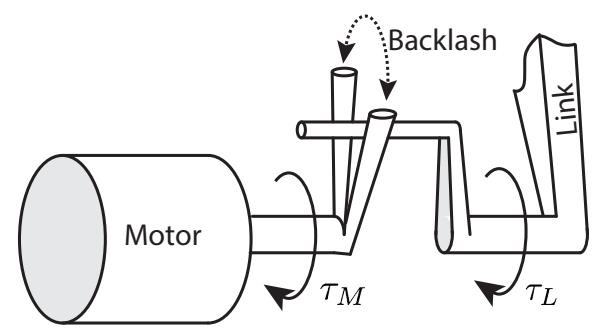

Fig. 1. Illustrative representation of a joint mechanism with backlash between motor and link.

effect of this play is that during motion the torque generated by the motor $\tau_{M}$ is not always transferred to the link. Hence, the toque acting on the link side $\tau_{L}$ is not equal to the motor torque.

It is straightforward to figure out that the backlash produces an error in the classical residual. This error is negligible when the backlash is very small, as commonly found in industrial robots; but it becomes significant with large joint backlash. To illustrate this effect, we simulated a single joint with a non negligible backlash controlled so as to generate a sinusoidal joint positioning. Figure 2 shows the classical residual obtained in this simulation. It is obvious that when the link moves inside the backlash gap, the residual detects the effects of a non existent external force. Basically, the residual follows the difference between the real torque applied to the link $\tau_{L}$ and the torque assumed in the residual computation $\tau_{M}$. To avoid this interpretation error, a measure of the link side torque would have been necessary. But this would require the use of a torque sensor.
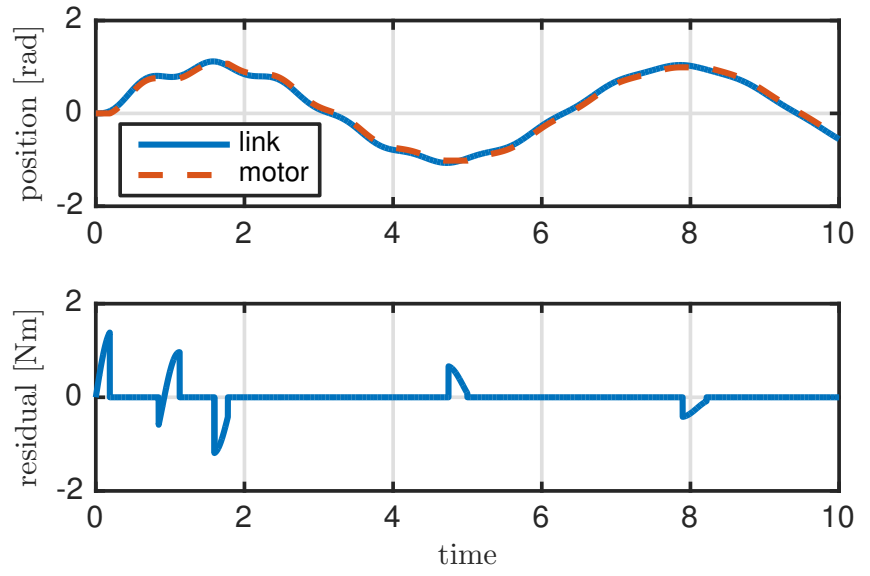

Fig. 2. Simulation of a joint with a 0.1 [rad] backlash: joint and motor position (top), and the obtained residual signal (bottom).

In the rest of the paper we propose a novel approach for using a residual based method in such a situation. The only additional sensor we require, with respect to the classical method, is for estimating the link side position (that can be obtained from another encoder, or -in our experiment casefrom the integration of the rotational speed velocity sensor). In fact, usually joint with large backlash are provided with two position sensors, one mounted on the motor side and the other one mounted on the link side. This is mandatory for controlling the joint position. Indeed, if only the motor position sensor had been present it would have been impossible to obtain a good link positioning, which usually is the desired output. On the other hand it would be really challenging, if not impossible, to guarantee the system stability using only a link side position feedback, see [9][10][11]. Therefore, we assume that a joint with large backlash, which is the target of the method we introduce, can provide two side position information (from sensors or estimators). This is the case of the Romeo arm that we use in the experiments (see Sec. V).

\section{Modeling AND IDENTIFICATION}

In the previous section we showed that the classical residual is not effective in the case of large backlash. To overcome this problem we need a proper modeling of this non-linear effects, and a method to identify their values.

\section{A. Backlash effects}

In literature there are different approaches for modeling backlash effects [12]. Here, we consider the dead-zone model, because it combines a good representation of the backlash behavior with a relative small model complexity (less prone to numerical errors and computation power). With reference to Fig. 3, $\alpha$ is the amplitude of the backlash zone and $\phi$ is the difference between the joint angle $q$ and the motor angle $\theta(\phi=q-\theta)$. The model considered combines three effects: i) when the link is inside the backlash gap $|\phi|<\alpha$, no torque is transferred from the motor to the link, i.e. $\tau_{L}=0$; otherwise ii) the motor torque is transferred to the link side, and iii) the contact with the border of the backlash gap is modeled as a spring damper system. 


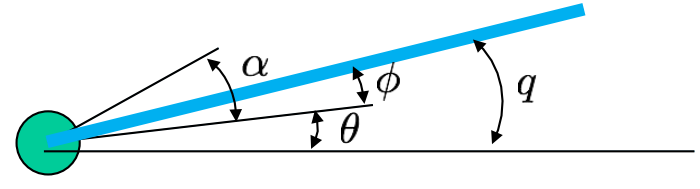

Fig. 3. Illustrative representation of the angles that play a role in the modeling of a joint with backlash.

Summing up

$$
\tau_{L}=\left\{\begin{array}{lcl}
0 & |\phi|<\alpha \\
K_{\phi p}(\phi+\alpha)+K_{\phi d} \dot{\phi}+\tau_{M} & \phi & \geq \alpha \\
K_{\phi p}(\phi-\alpha)+K_{\phi d} \dot{\phi}+\tau_{M} & \phi & \leq-\alpha
\end{array}\right.
$$

where $K_{\phi p}$ and $K_{\phi d}$ represent respectively the stiffness and the damping of the contact.

\section{B. Friction effects}

Beside the backlash effect, the other non-linear behavior that has to be taken into account is friction. When the link moves together with the motor $(|\phi| \geq \alpha)$, friction composed of viscous and static terms is assumed,that is $K_{\theta v} \dot{\theta}+K_{\theta s} \operatorname{sign}(\dot{\theta})$. Here, $K_{\theta v}$ is the viscous friction coefficient, and $K_{\theta s}$ is the static friction coefficient. Differently, when the link moves inside the backlash gap $(|\phi|<\alpha)$ two friction effects have to be considered: i) the friction that opposes to the relative rotation between the motor and the link $K_{\phi v} \dot{\phi}+K_{\phi s} \operatorname{sign}(\dot{\phi})$, and ii) the friction action on the link motion $K_{q v} \dot{q}+K_{q s} \operatorname{sign}(\dot{q})$. Where $K_{\phi v}$ and $K_{q v}$ are the viscous friction coefficients, and $K_{\phi s}$ and $K_{q s}$ are the static friction coefficients.

Summing up

$$
\tau_{\mu}=\left\{\begin{array}{cr}
K_{\phi v} \dot{\phi}+K_{\phi s} \operatorname{sign}(\dot{\phi})+ & \\
K_{q v} \dot{q}+K_{q s} \operatorname{sign}(\dot{q}) & |\phi|<\alpha \\
K_{\theta v} \dot{\theta}+K_{\theta s} \operatorname{sign}(\dot{\theta}) & \text { otherwise }
\end{array}\right.
$$

\section{Parameter estimation}

Considering both the backlash and friction effects acting on all $n$ joints of the robot, the dynamic model is

$$
M \ddot{\boldsymbol{q}}+\boldsymbol{c}(\boldsymbol{q}, \dot{\boldsymbol{q}})+\tau_{\mu}=\tau_{L}+\tau_{\mathrm{ext}}
$$

where, the vectors $\tau_{\mu}$ and $\tau_{L}$ contain respectively the friction contribution and the link side torque for the joints. Assuming the knowledge of kinematic parameters and a reliable estimation of link masses, center of mass and inertia, as this is indeed required even in the classical residual, we need to estimate the non-linear parts. Basically, we need to estimate the backlash gap $\alpha$, the backlash contact coefficients $K_{\phi p}$ and $K_{\phi d}$, and the friction coefficients $K_{\phi s}, K_{\phi v}, K_{q s}$, $K_{q v}, K_{\theta s}$ and $K_{\theta v}$.

An approach for estimating both the backlash and the friction effects was presented in [13]. The method was based on controlling the joint with a number of trapezoidal motor velocities profiles, using the part in which the velocity is constant to estimate the friction, and the part where the velocity switches sign to estimate the backlash gap. This is based on the idea that the link moves from one side of the backlash gap to the other side only when the velocity changes sign. Unfortunately, this assumption is correct only if the joint rotational axis is perpendicular to gravity, i.e. $g(q)=0 \quad \forall q \in \mathbb{R}$. In general, it is possible that the link remains in the same side of the backlash gap when the velocity changes sign or when it jumps to the other side even with constant velocity. In [14] a second order sliding mode observer is presented, but the backlash effects are considered decoupled with respect to friction effects, and friction is assumed known a priori. The same simplistic assumption is necessary in [15], where a State Augmented Extended Kalman Filter is used. Isolated and decoupled backlash and friction behaviors are assumed also in [16], but in that work the friction effect is estimated. A non-linear regressors, based on a peculiar backlash model is presented in [17], but it has been developed considering an isolated backlash system; thus, without considering root dynamic and friction.

Our approach is based on two parts, in the first part we estimate the backlash gap size $\alpha$, and in the second part, we estimate all the other coefficients. This is the only realistic decoupling, since the backlash gap acts at the position level, while all other effects act on the joint torque.

To estimate the gap we servo the motor to a static position with high stiffness (large proportional gain). Then we manually move the link inside the backlash gap, avoiding to strain the contact. Then, the backlash gap is evaluated by observing the range of values of $q-\theta$. A more rigorous method, although more complicated, was presented in [12], where the frequency domain response of the system is taken into account. Alternatively, [18] performed a pre-estimation based on observing the switching instants, and a refining method trough least squares minimization.

For all the remaining coefficients we used an estimation approach based on a variation of the classical residual. Consider a robot motion without external contacts $\left(\boldsymbol{\tau}_{\text {ext }}=\mathbf{0}\right)$, the contribution of all un-modeled non-linear effects can be extrapolated by using the residual

$\boldsymbol{r}_{n l}(t)=K r\left[\boldsymbol{M} \dot{\boldsymbol{q}}-\int_{0}^{t}\left(\overline{\boldsymbol{\tau}}_{M}+\boldsymbol{C}^{T} \dot{\boldsymbol{q}}-\boldsymbol{g}+\boldsymbol{r}_{n l}(s)\right) d s\right]_{(8)}$

where $\bar{\tau}_{M}=\left[\bar{\tau}_{M, 1} \ldots \bar{\tau}_{M, n}\right]^{T}$, with

$$
\bar{\tau}_{M, i}= \begin{cases}0 & \left|\phi_{i}\right|<\alpha_{i} \\ \tau_{M, i} & \text { otherwise }\end{cases}
$$

As in the classical residual (2), it is simple to prove that $\boldsymbol{r}_{n l}$ is a first order filter of the non-linear contribution $\bar{\tau}_{L}-\tau_{\mu}$, where $\overline{\boldsymbol{\tau}}_{L}=\boldsymbol{\tau}_{L}-\overline{\boldsymbol{\tau}}_{M}$.

For each sample time $k$ we collect the joint and motor position $\boldsymbol{q}^{k}$ and $\boldsymbol{\theta}^{k}$, the residual $\boldsymbol{r}_{n l}^{k}$. At the end of the experiment we compute $\phi^{k}=\boldsymbol{q}^{k}-\boldsymbol{\theta}^{k}$, and we derive the velocities $\dot{\boldsymbol{q}}^{k}, \dot{\boldsymbol{\theta}}^{k}$ and $\dot{\boldsymbol{\phi}}^{k}$.

Off-line, for each joint $i$ we group $\boldsymbol{R}_{n l, i}=$ 


$$
\begin{gathered}
{\left[r_{n l, i}^{1}, r_{n l, i}^{2}, \ldots, r_{n l, i}^{p}\right]^{T} \text { and } \boldsymbol{A}_{i}=\left[\boldsymbol{A}_{i}^{1}, \boldsymbol{A}_{i}^{2}, \ldots, \boldsymbol{A}_{i}^{p}\right]^{T}, \text { with }} \\
\boldsymbol{A}_{i}^{k}=\left\{\begin{array}{l}
{\left[\dot{\phi}_{i}^{k}, \operatorname{sign}\left(\dot{\phi}_{i}^{k}\right), \dot{q}_{i}^{k}, \operatorname{sign}\left(\dot{q}_{i}^{k}\right), \mathbf{0}\right]^{T} \quad\left|\phi_{i}^{k}\right|<\alpha_{i}} \\
{\left[\mathbf{0},\left(\dot{\phi}_{i}^{k}+\alpha_{i}\right), \dot{\phi}_{i}^{k}, \dot{\theta}_{i}^{k}, \operatorname{sign}\left(\dot{\theta}_{i}^{k}\right)\right]^{T} \quad \phi_{i}^{k} \geq \alpha_{i}} \\
{\left[\mathbf{0},\left(\dot{\phi}_{i}^{k}-\alpha_{i}\right), \dot{\phi}_{i}^{k}, \dot{\theta}_{i}^{k}, \operatorname{sign}\left(\dot{\theta}_{i}^{k}\right)\right]^{T} \quad \phi_{i}^{k} \leq-\alpha_{i}}
\end{array}\right.
\end{gathered}
$$

where $p$ is the number of the collected samples.

At this point, all non-linear coefficients for joint $i$ are estimated as

$$
\begin{aligned}
& {\left[\hat{K}_{\phi v, i}, \hat{K}_{\phi s, i}, \hat{K}_{q v, i}, \hat{K}_{q s, i}, \hat{K}_{\phi p, i},\right.} \\
& \left.\quad \hat{K}_{\phi d, i}, \hat{K}_{\theta v, i}, \hat{K}_{\theta s, i}\right]^{T}=\hat{\boldsymbol{K}}_{i}=\boldsymbol{A}_{i}^{\#} \boldsymbol{R}_{n l, i}
\end{aligned}
$$

where $\#$ is the symbol of the Moore-Penrose pseudo inverse. If the executed motion excites sufficiently the joint $i$, eq. (11) is a reliable estimation of the coefficients characterizing the joint backlash and friction behaviors.

\section{IMPLEMENTING THE RESIDUAL}

In the previous section we have defined a dynamic model that takes into account backlash and friction effects (7), and we proposed a novel approach to estimate the coefficients that characterize its behavior. Reaching this point, it is straightforward to derive a new residual for estimating joint torques due to external contacts $\tau_{\text {ext }}$,

$$
\begin{aligned}
\boldsymbol{r}_{b s}(t)= & K_{b s}[\boldsymbol{M} \dot{\boldsymbol{q}}- \\
& \left.\int_{0}^{t}\left(\dot{\boldsymbol{M}} \dot{\boldsymbol{q}}+\boldsymbol{\tau}_{L}-\boldsymbol{\tau}_{\mu}-\boldsymbol{c}(\boldsymbol{q}, \dot{\boldsymbol{q}})+\boldsymbol{r}_{b s}(s)\right) d s\right]
\end{aligned}
$$

It follows trivially that $\dot{\boldsymbol{r}}_{b s}=K_{b s}\left[\boldsymbol{\tau}_{\text {ext }}-\boldsymbol{r}_{b s}\right]$, proving that this novel residual is a first order filter of the joint torque due to external contacts.

Note that in our implementation we did not factorize $\boldsymbol{c}(\boldsymbol{q}, \dot{\boldsymbol{q}})=\boldsymbol{C} \dot{\boldsymbol{q}}+\boldsymbol{g}(\boldsymbol{q})$ and used directly $\dot{\boldsymbol{M}}$. In fact, it has been shown in [19] that, thanks to the use of the spatial algebra [20], these values can be obtained with a computational complexity smaller than the method to compute $\boldsymbol{C}$ proposed in [21]. In fact, in [21] the factorized centrifugal and Coriolis term $\boldsymbol{C}$ are computed with a modified version of the NewtonEuler method, that has complexity $\mathcal{O}\left(n^{2}\right)$, since $n$ instances of the Newton-Euler routine needs to be called. While, the method we proposed in [19] is of $\mathcal{O}(n)$ complexity.

A discrete-time implementation of the residual $r_{b s}(k)=$ $r_{b s}\left(t_{k}\right)$ at $t=t_{k}=k T$ is obtained using Tustin rule in (12), yielding to

$$
\begin{aligned}
\boldsymbol{I}_{\tau}(k) & =\boldsymbol{I}_{\tau}(k-1)+\frac{\boldsymbol{\tau}_{b s}(k)+\boldsymbol{\tau}_{b s}(k-1)}{2} T \\
\boldsymbol{\tau}_{b s}(k) & =\dot{\boldsymbol{M}}(k) \dot{\boldsymbol{q}}(k)+\boldsymbol{\tau}_{L}(k)-\boldsymbol{\tau}_{\mu}(k)-\boldsymbol{c}(\boldsymbol{q}, \dot{\boldsymbol{q}}, k) \\
\overline{\boldsymbol{r}}(k) & =K_{b s}\left(\boldsymbol{M}(k) \dot{\boldsymbol{q}}(k)-\boldsymbol{I}_{\tau}(k)\right) \\
\boldsymbol{r}_{b s}(k) & =\frac{2-T K_{b s}}{2+T K_{b s}} \boldsymbol{r}_{b s}(k-1)+\frac{2(\overline{\boldsymbol{r}}(k)-\overline{\boldsymbol{r}}(k-1))}{2+T K_{b s}},
\end{aligned}
$$

where $T$ is the periodic sampling time.

\section{EXPERIMENTAL RESULTS}

To assess and show the effectiveness of our presented methodology, we report a series of experimental results. The experiments are executed on the left arm of the Romeo robot ${ }^{2}$ provided by SoftBank Robotics. It consists of a seven degree of freedom robotic arm plus an actuated hand (Fig. 4). The information available for each joint are: i) motor current $c$, from which we obtain the motor torque, knowing the current to torque constant $K_{c}$ provided by the motor manufacturer: $\tau_{M}=K_{c} c$; ii) joint position $q$, and iii) motor velocity $\dot{\theta}$, that we numerically integrate to estimate the the motor position $\theta$, knowing the initial motor position. The nominal dynamic model can be obtained by using the kinematic and dynamic information that are freely available and accessible ${ }^{3}$. The sampling time used in the experiments is 10 [ms].

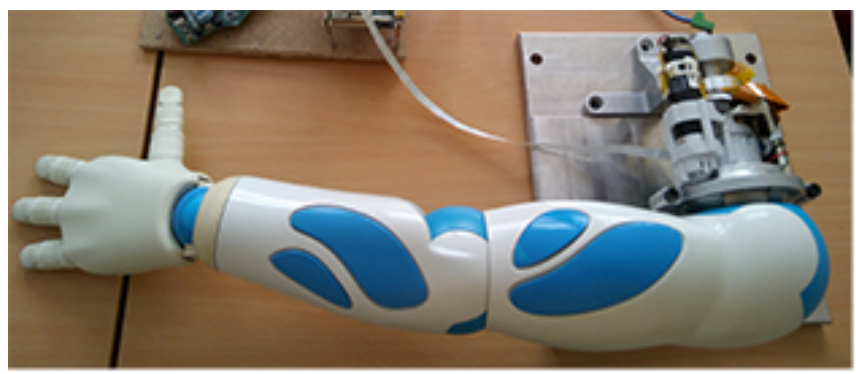

Fig. 4. The prototype of the left arm of Romeo available in our laboratory.

\section{A. System identification}

As discussed in Sec. III, the backlash and friction parameters have to be estimated. The first parameters is the backlash gap size $\alpha$. Following the procedure described in Sec. III-C, we servo the robot to remain at a fixed static position, and then we manually moved each link, softly hitting the border of the backlash gap. Figure 5 shows the difference between the link and the motor angle obtained for joint 2 (shoulder roll), which is the joint with the larger backlash in the robot's $\operatorname{arm}\left(\alpha_{2} \approx 0.025\right.$ [rad]). The complete list of the estimated backlash gap size for all joints is

$$
\hat{\boldsymbol{\alpha}}=[0.018,0.025,0.003,0.011,0,0,0.023][\mathrm{rad}] .
$$

In the second part of the identification procedure, the robot is controlled using the trajectory reported in Fig. 6. The residual (8) is used to estimate all un-modeled effects, with $K_{r}=100$. As described in Sec. I, the residual gain $K_{r}$ sets the bandwidth of the filter action given by the residual. Assuming that all un-modeled effects are due to backlash and friction, the coefficients that characterize this two effects are identified using (11).

The Fig.7 shows the obtained residual, compared with the behavior of backlash and friction reconstructed with the estimated parameters

$$
\hat{\boldsymbol{r}}_{n l, i}=\boldsymbol{A}_{i} \hat{\boldsymbol{K}}_{i}
$$

\footnotetext{
${ }^{2}$ http: //projetromeo.com/

${ }^{3}$ http://projetromeo.com/sites/default/files/ romeo-documentation/
} 


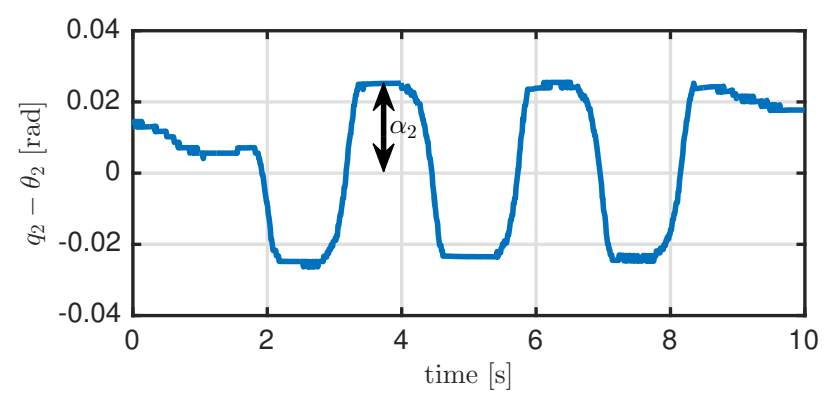

Fig. 5. Difference between link and motor position during the experiment for the estimation of the backlash gap size of the second joint.

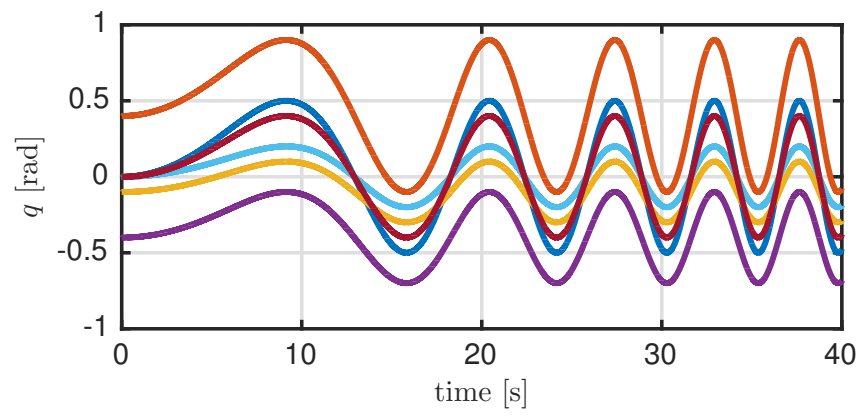

Fig. 6. Joint trajectories used for estimating backlash and friction coefficients.

The match is quite good but not 'perfect', i.e. it can certainly

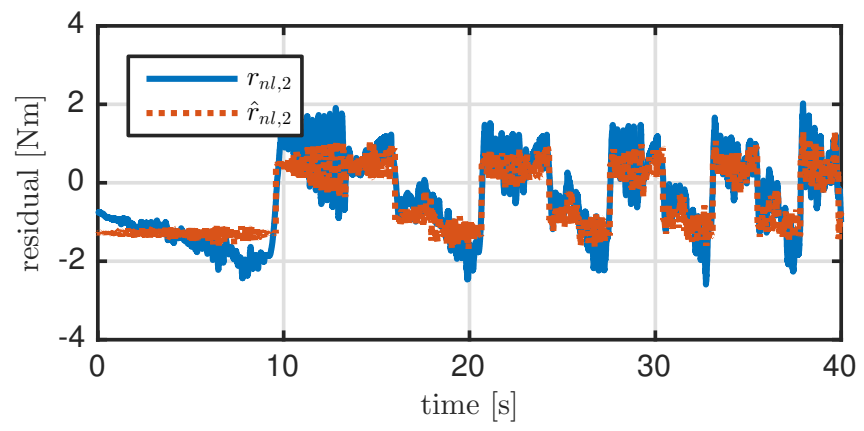

Fig. 7. Residual estimating the nonlinear behavior of the robot (blue) and reconstructed behavior using the estimated parameters (red).

be improved. Even trying to move the robot with different trajectories or using different models for the backlash and friction, we did not observe a significant improvement in the identification results. Thus, we attribute the remaining error to the fact that we are using the nominal dynamic parameters (masses, inertia, center of mass) given by the manufacturer from the CAD modeling. Hence, it is the same for all the robots they build and distribute, which very likely do not match perfectly with the one we have in hands. However, the results obtained are sufficiently accurate for our application, as it will be shown in the next sections.

\section{B. Motion without external forces}

In the first experiment, the robot is controlled with a sinusoidal motion for the first joint, while the other joints are maintained at their respective initial zero-position. During the motion, no external forces are applied, therefore a residual close to zero is expected. The residual (12), compared
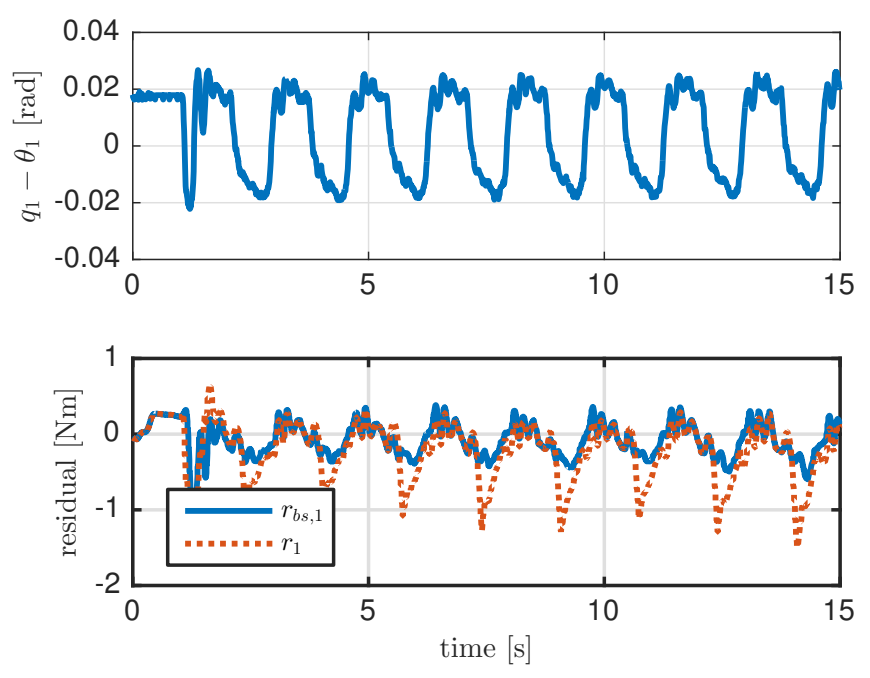

Fig. 8. Comparison between the classical residual (bottom red) and our enriched residual (bottom blue). The behavior of the difference between motor and link angle, characterizing the backlash, is presented in the top plot.

with the classical residual (2) obtained in the experiment is reported in Fig. 8. It is evident that when the link move from one side to the other of the backlash gap $\left(q_{1}-\theta_{1}\right.$ passes trough 0 ), the classical residual has a large error. This reduces the possible use of the classical residual to detect collision. While, for our proposed residual, a small threshold is able to filter the error due to inaccuracies in the dynamic model.

\section{Detecting contact}

In the second experiment we show the effectiveness of our residual method to estimate the joint torque due to external contact forces. We perform the test by exerting external forces in different points of the robot, both with the robot at rest (static) and in motion (dynamics). The robot is at rest for the first 20 seconds, and then it moves with sinusoidal motion for joints 1, 3, 4 and 7, as represented in Fig. 9. In Fig. 10 screen shots of the experiment shows the times

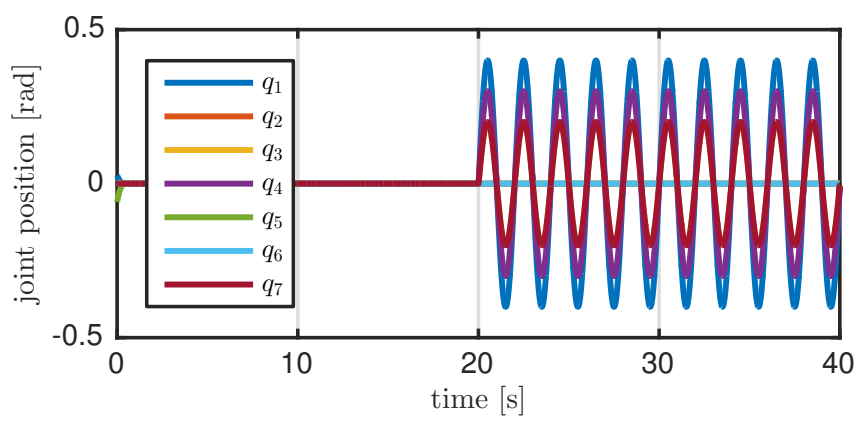

Fig. 9. Desired robot trajectory used in the second experiment: in the first part the robot remains in the initial configuration, successively, sinusoidal motion is commanded for $q_{1}, q_{3}, q_{4}$ and $q_{7}$.

the external forces are applied to the robot. Threshold is used to filter the residual, so as to get the filtered residual 

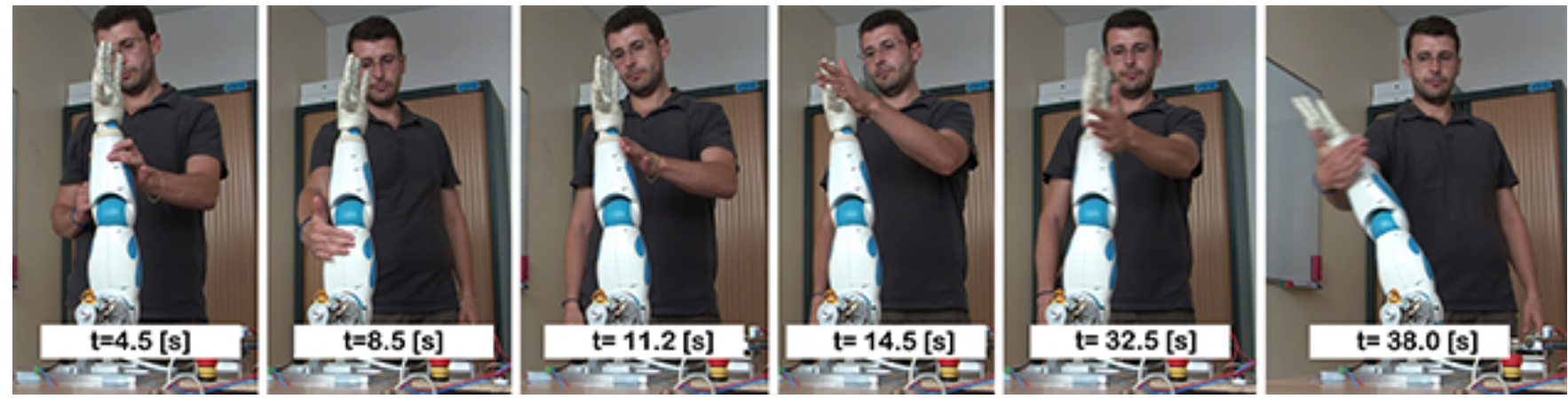

Fig. 10. Screen shots from the second experiment and the timings an external force is applied to the robot are represented.

$\overline{\boldsymbol{r}}_{b s}$ plotted in Fig. 11. In the experiment, the different
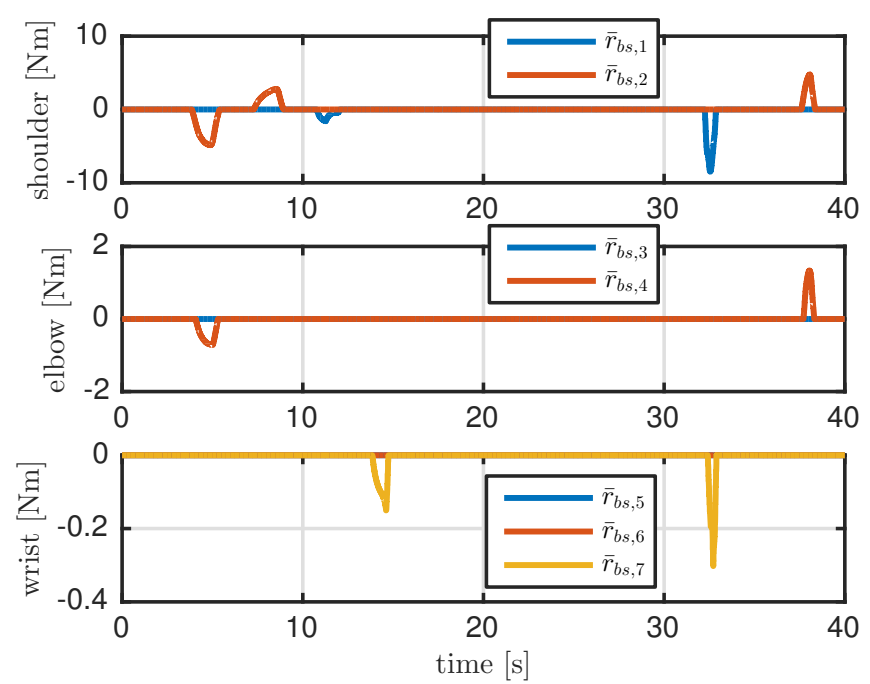

Fig. 11. Estimated joints torque due to external contacts (see Fig. 10). All contact are detected without false positive, even during robot motion.

characteristic of each joint is considered by using a different threshold for each joint. The threshold values used are $[0.8,0.5,0.4,0.3,0.2,0.2,0.2][\mathrm{Nm}]$.

It is easy to check that with our presented residual all contacts are correctly detected and no false positive occurred, even when the robot is moving. Considering the results reported in the previous section, shown in Fig. 8, it is obvious that false positive would have been detected by the classical residual every time the links moves within the backlash gap.

\section{Human-robot physical collaboration}

In the last experiment we tested the use of the presented residual signal to physically interact with the robot. Such a functionality is needed in many aspects of human-robot interaction, e.g. in physically guided teaching, to implement safe compliant behavior, etc. To this end, the residual signal can be used to generate an admittance control, in which the force exerted on the robot is transformed to a desired joint velocity, according to the law (more sophisticated ones are of course possible)

$$
\dot{\boldsymbol{q}}_{d}=K_{a} \boldsymbol{r}_{b s} .
$$

The robot is asked to track a sinusoidal desired motion for the fist joint, while all other joints remain in the current configuration. When a contact is detected $\left(\left\|\overline{\boldsymbol{r}}_{b s}\right\|>0\right)$, the robot follows the admittance law (15), and the new configuration is then manually guided by the human (sustained physical interaction). When the residual remains below the threshold for at least 2 seconds, the sinusoidal motion starts again from the new configuration. Screen shots the third experiment are presented in Fig. 12. The robot is executing the sinusoidal motion (left); Then the human physically interact with the robot, and, thanks to the presented residual signal, he moves the robot to a new configuration interactively (center); The robot starts the sinusoidal motion from the new configuration (right). The joint position motion, and the residual $\overline{\boldsymbol{r}}_{b s}$ observed in this experiment are plotted in Fig. 13. To better appreciate the effectiveness of the presented approach, the complete sequence of the second and third experiments is reported in the accompanying video.

\section{CONCLUSIONS}

In this paper we presented an extension of the generalized momentum-based residual for estimating external contact forces when they occur on low cost personal robots. In particular we considered the two main common non-linear effects present in the joint of these robots, backlash and friction. Using a model that integrates backlash and friction in the dynamic equation, we devised an identification approach based on a modified residual. Thanks to this estimation we were able to build a novel residual expression to estimate joints torque due to external contact forces. We showed that this residual can be used to provide to low cost personal robots the capabilities for detecting contacts and also to physically interact with it through e.g. manual guidance. Our results can be applied to a wide spectrum of social robotics applications, namely those where low cost robots are to be manipulated by touch.

As future work, we will examine some remaining technical aspects: For instance, it could be interesting to devise a way to automate the thresholding and the tuning of the gain of the residual (the gain can be adaptive or learned and configuration dependant). We also plan to integrate the residual approach in a coupled way using the motor current when available (redundancy). Finally, we will investigate 

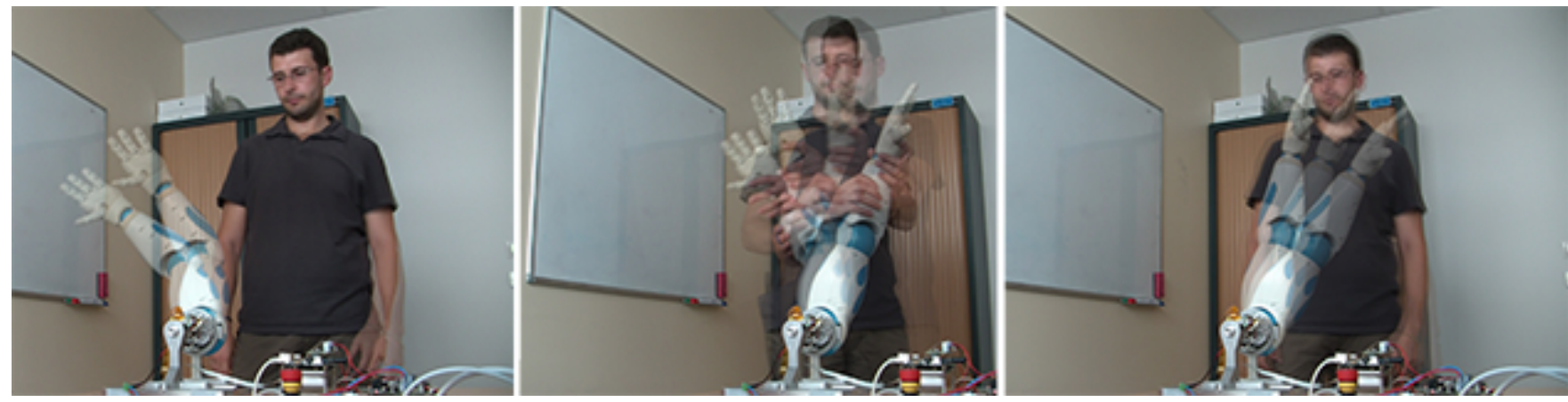

Fig. 12. Screen shots from the third experiment. The robot executes the desired sinusoidal motion with the first link (left). The human drives the robot to a new configuration by interacting physically with the robot (center). The robot starts again the sinusoidal motion from the new configuration (right).
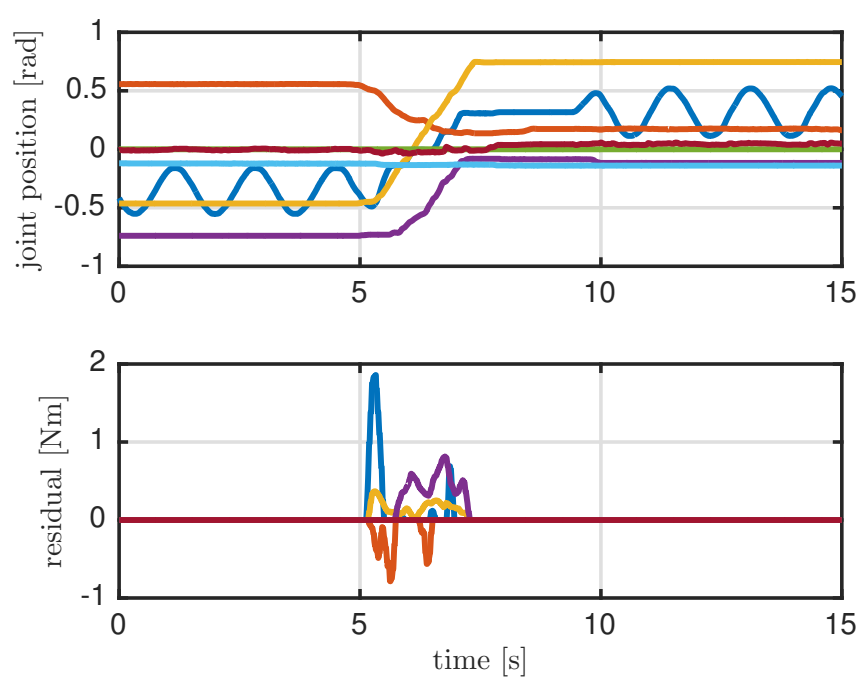

Fig. 13. Joint position (top) and residual signal (bottom), obtained during the third experiment.

the residual method in the presence of link 's soft-covers instead of the current rigid ones. Finally, we could also use the residual to improve the robot inertia parameters identification [22].

\section{REFERENCES}

[1] B. Gates, "A robot in every home," Scientific American, vol. 295, no. 1, pp. 58-65, 2007.

[2] P. Dario, E. Guglielmelli, and C. Laschi, "Humanoids and personal robots: Design and experiments," Journal of Robotic Systems, vol. 18, no. 12, pp. 673-690, 2001.

[3] K. A. Wyrobek, E. H. Berger, H. F. M. Van der Loos, and J. K. Salisbury, "Toward a personal robotics development platform: Rationale and design of an intrinsically safe personal robot," in IEEE International Conference on Robotics and Automation, 2008, pp. 2165-2170.

[4] J. Lafaye, D. Gouaillier, and P. B. Wieber, "Linear model predictive control of the locomotion of pepper, a humanoid robot with omnidirectional wheels," in IEEE-RAS International Conference on Humanoid Robots, 2014, pp. 336-341.

[5] A. De Luca, A. Albu-Schäffer, S. Haddadin, and G. Hirzinger, "Collision detection and safe reaction with the DLR-III lightweight robot arm," in IEEE/RSJ International Conference on Intelligent Robots and Systems, 2006, pp. 1623-1630.

[6] M. Geravand, F. Flacco, and A. De Luca, "Human-robot physical interaction and collaboration using an industrial robot with a closed control architecture," in IEEE International Conference on Robotics and Automation, 2013, pp. 4000-4007.
[7] E. Magrini, F. Flacco, and A. De Luca, "Estimation of contact forces using a virtual force sensor," in IEEE/RSJ Internationa Conference on Intelligent Robots and Systems, 2014, pp. 2126-2133.

[8] E. Magrini, F. Flacco, and A. De Luca, "Control of generalized contact motion and force in physical human-robot interaction," in IEEE International Conference on Robotics and Automation, 2015, pp. 2298-2304.

[9] M. Nordin and P.-O. Gutman, "Controlling mechanical systems with backlash: a survey," Automatica, vol. 38, no. 10, pp. 1633 - 1649, 2002.

[10] L. Marton and B. Lantos, "Control of mechanical systems with stribeck friction and backlash," Systems \& Control Letters, vol. 58, no. 2, pp. $141-147,2009$.

[11] B.-J. Jung, J.-S. Kong, B.-H. Lee, S.-M. Ahn, and J.-G. Kim, "Backlash compensation for a humanoid robot using disturbance observer," in 30th Annual Conference of the IEEE Industrial Electronics Society, vol. 3, 2004, pp. 2142-2147.

[12] M. Nordin, P. Bodin, and P.-O. Gutman, New Models and Identification Methods for Backlash and Gear Play. Springer London, 2001, pp. $1-30$

[13] L. Marton and B. Lantos, "Friction and backlash measurement and identification method for robotic arms," in Advanced Robotics, 2009. ICAR 2009. International Conference on, 2009, pp. 1-6.

[14] R. Merzouki, J. Davila, L. Fridman, and J. Cadiou, "Backlash phenomenon observation and identification in electromechanical system," Control Engineering Practice, vol. 15, no. 4, pp. 447 - 457, 2007.

[15] G. Hovland, S. Hanssen, E. Gallestey, S. Moberg, T. Brogardh, S. Gunnarsson, and M. Isaksson, "Nonlinear identification of backlash in robot transmissions," in 33rd International Symposium on Robotics. Citeseer, 2002.

[16] M. Ruderman, F. Hoffmann, and T. Bertram, "Modeling and identification of elastic robot joints with hysteresis and backlash," IEEE Transactions on Industrial Electronics, vol. 56, no. 10, pp. 3840-3847, 2009

[17] J. Vrs, "Modeling and identification of systems with backlash," Automatica, vol. 46, no. 2, pp. $369-374,2010$.

[18] L. Ravanbod-Shirazi and A. Besanon-Voda, "Backlash identification a two step approach," IFAC World Congress Proceedings Volumes, vol. 35, no. 1, pp. $85-90,2002$.

[19] F. Flacco, A. Paolillo, and A. Kheddar, "Residual-based contacts estimation for humanoid robots," in IEEE-RAS International Conference on Humanoid Robots, Cancun, Mexico, 15-17 November 2016.

[20] R. Featherstone, Rigid Body Dynamics Algorithms. Secaucus, NJ, USA: Springer-Verlag New York, Inc., 2008.

[21] A. D. Luca and L. Ferrajoli, "A modified newton-euler method for dynamic computations in robot fault detection and control," in IEEE International Conference on Robotics and Automation, 2009, pp. $3359-3364$.

[22] J. Jovic, A. Escande, K. Ayusawa, E. Yoshida, A. Kheddar, and G. Venture, "Humanoids and humans inertia parameters identification using hierarchical optimization," IEEE Transactions on Robotics, vol. 32, no. 3, pp. 726-735, 2016. 